Research in Astronomy and Astrophysics manuscript no.

(LTEX: ms raa.tex; printed on June 28, 2021; 8:14)

\title{
Mass flow in the circumbinary disk with gap around supermassive binary black holes
}

\author{
Ning-Yu Tang ${ }^{1}$ and Ye-Fei Yuan ${ }^{1}$ \\ Key Laboratory for Research in Galaxies and Cosmology CAS,Department of Astronomy, University of \\ Science and Technology of China, Hefei, Anhui, 230026, China; yfyuan@ustc.edu.cn
}

\begin{abstract}
In this paper, we study the interaction between the supermassive binary black holes in elliptical orbit and their surrounding disk with a gap. The gap in the disk is a low density region formed due to the tidal effects of the less massive black hole. The binary we have investigated has a sub-parsec separation and is coplanar with the disk. We find that the maximum variation of the surface density in the gap reaches $50 \%$ during an orbital period. However, in other regions of the disk, the density variation is much less than $1 \%$. Furthermore, we calculate the corresponding variation of spectral energy distribution within a period, but little variation is found. The reason for these results is that the viscosity timescale of the disk at the binary radius is much longer than the orbital period of the binary.
\end{abstract}

Key words: binaries:close — accretion disks — methods: numerical

\section{INTRODUCTION}

Supermassive binary black holes (SMBBHs) is believed to form during galaxy mergers which happens frequently in the universe according to the hierarchical models of galaxy formation. It is widely accepted that the merging of the two SMBHs would go through three stages (Begelman et al. 1980, Colpi et al. 2011). The standard picture is as follows. After the initial phase of merging of the galactic cores due to the dynamical friction, the two SMBHs would form a bound pair. The second phase in which the SMBBHs hardens via 3-body scattering off single star is still uncertain. It is of theoretical difficulty to shrink the separation of a SMBBHs by a factor of $\sim 100$ after its formation at a separation of $\sim 1 \mathrm{pc}$ ( Merritt et al. 2002). This is known as the "final parsec problem". At the final phase, when SMBBHs reaches a separation of $\sim 0.01 \mathrm{pc}$, the two black holes will coalesce within a relatively short time interval through emission of gravitational waves (GWs) (Sathyaprakash \& Schutz et al. 2009). Measuring the separation distribution between pairs of SMBHs helps to constrain the timescales of the different stages of SMBHs merging, and to estimate the number of gravitational wave sources that can be detected with the Laser Interferometer Space Antenna (LISA) in one year (Dotti et al.2006).

Currently, there is no direct detection of GWs, the search for SMBBHs mainly focus on the first and 
SMBBHs, for instance, NGC6240 (Komossa et al. 2003), COSMOS J100043.15+020637.2 (Comerford et al.2009) and J0402+379 (Rodriguez et al.2006), have been identified spatially in a single galaxy via X-ray, optical and radio imaging spectroscopy. A few galaxies which contain double-peaked [O III] or H $\beta$ lines with velocity offsets are suggested as pairs of AGNs ( Zhou et al. 2004, Dotti et al.2009; Wang et al.2009, Shen et al.2010, Liu et al. 2010). In addition, several other ways have been proposed to identify SMBBHs: X-shaped morphology of radio lobes (Merritt et al. 2002), double-double radio galaxies (Liu et al. 2003), orbital motion of the compact core with a periodic flux variation (Sudou et al. 2003), periodic optical and radio outbursts (e.g., OJ 287) (Valtonen et al.2008), and so on.

Eccentricity of SMBBHs in merging galaxies with large amount of gas is proved to be high (Roedig et al. 2011), which leads to periodic accretion flows onto the SMBH and then periodic occurrence of peaked flares in light curves (Hayasaki et al.2007). Hayasaki (2012) carried out the smooth particle hydro-dynamics simulation on circumbinary disk with a central cavity and calculated the periodic mass accretion rate $\dot{M}$ onto the SMBHs. The corresponding periodic luminosity (luminosity $L=\eta \dot{M} c^{2}$ ) is believed as the indicator of SMBBHs at sub-parsec separation. But the mass accretion rate in their work is defined as the mass rate falling into an fixed "accretion" sphere without considering the detailed accretion physics. The radius of the fixed "accretion" sphere is chosen to be $0.1 a$, where $a$ is the semi-major axis of the binary orbit.

In this paper, the matter is accreted through a disk existing around the primary $\mathrm{SMBH}$, rather than a defined "accretion" sphere. The matter across the gap flows onto the inner disk and is accreted into the primary SMBH by viscosity effect. This is a more realistic and physical consideration.

The outline of this paper is as follows. In section 2, we introduce the basic physics of binary system, followed by the description of the simulation in section 3. Finally, results and discussions are presented in section 4.

\section{PHYSICAL MODEL OF THE ACCRETION IN SMBBHS}

\subsection{Basic equations governing circumbinary disk}

It is assumed that the secondary SMBH exists in the accretion disk of the primary SMBH and its orbital plane is coplanar with the disk plane (Bogdanovic et al. 2007, Dotti et al. 2010). The dynamics of this system is similar to that of the protoplanetary disk in the preliminary formation of a star. The latter has been widely discussed in the past decades (Goldreich et al. 1980, Takeuchi et al. 1996, Espaillat et al. 2008). The hydrodynamic equations of the evolution of the proto-planetary disk are described as follows (Kley et al. 1999; Crida et al. 2009):

$$
\begin{gathered}
\frac{\partial \Sigma}{\partial t}+\nabla \cdot(\Sigma \mathbf{u})=0 \\
\frac{\partial(\Sigma v)}{\partial t}+\nabla \cdot(\Sigma v \mathbf{u})=\Sigma r(\omega+\Omega)^{2}-\frac{\partial p}{\partial r}-\Sigma \frac{\partial \Phi}{\partial r}+f_{r}, \\
\frac{\partial\left[\Sigma r^{2}(\omega+\Omega)\right]}{\partial t}+\nabla \cdot\left[\Sigma r^{2}(\omega+\Omega) \mathbf{u}\right]=-\frac{\partial p}{\partial \varphi}-\Sigma \frac{\partial \Phi}{\partial \varphi}+f_{\varphi},
\end{gathered}
$$

These equations are shown in cylindrical coordinates $(r, \varphi, z)$, where $r$ is the radial coordinate, $\varphi$ is the azimuthal angle, $z$ is the vertical axis. The reference frame rotates with the angular velocity $\Omega$. The rotation 
Equation (1) represents the conservation of mass. $\Sigma$ denotes the surface density and $\mathbf{u}$ is the velocity vector of the fluid. Equation (2), (3) represent the momentum equation in $r$ and $\varphi$ direction. The symbol $v=u_{r}$ means radial velocity and $\omega=u_{\varphi} / r$ means the angular velocity of the flow, both of which are measured in the corotating frame. $p$ is the vertically integrated (two-dimensional) pressure. The gravitational potential $\Phi$ is contributed by both the primary SMBH with mass $M_{p}$ and the secondary BH with mass $M_{s}$, which is given by

$$
\Phi=-\frac{G M_{p}}{\left|\mathbf{r}-\mathbf{r}_{p}\right|}\left(1+\frac{M_{s}\left|\mathbf{r}-\mathbf{r}_{p}\right|}{M_{p}\left|\mathbf{r}-\mathbf{r}_{s}\right|}\right)=\Phi_{0}(1+\delta),
$$

where $G$ is the gravitational constant and $\mathbf{r}_{p}, \mathbf{r}_{s}$ are the position vectors of the primary and secondary $\mathrm{BH}$, respectively. $\Phi_{0}$ is the gravitational potential induced by the primary SMBH and $\delta$ represents the potential perturbations induced by the secondary BH. If $\delta$ is small, Fourier decomposition of the timeperiodic gravitational potential of the binary is applicable. The potential is given by a double series

$$
\Phi(r, \theta, t)=\sum_{m l} \phi_{m l}(r) e^{\left[i\left(m \theta-l \Omega_{B} t\right)\right]},
$$

where $l$ is the time-harmonic number, $m \geq 0$ is the azimuthal number, $\phi_{m l}(r)$ is the radially variable potential component,

$$
\phi_{m l}=\frac{1}{2 \pi^{2}} \int_{0}^{2 \pi} \int_{0}^{2 \pi} \Phi \cos \left(m \theta-l \Omega_{B} t\right) d \theta d\left(\Omega_{B} t\right)
$$

and $\Omega_{B}=\left(G M / a^{3}\right)^{1 / 2}$ is the mean motion of the binary, with $M$ and $a$ denoting the total mass and semimajor axis of the binary, respectively (Artymowicz et al.1994). Two terms in equation (2)-(3), $\frac{\partial \Phi}{\partial r}$ and $\frac{\partial \Phi}{\partial \varphi}$, act as tidal effects on the disk. $f_{r}$ and $f_{\varphi}$ represent the viscous force per unit area acting in the radial and azimuthal direction. The explicit forms of $f_{r}$ and $f_{\varphi}$ are given by Kley (1999).

\subsection{Resonance}

Artymowicz (1994) had analytically discussed the gravitational interaction of a generally eccentric binary star system with the circumbinary gaseous disks. The interaction between the circular binary and the circumbinary disk can occur via $(m, l)=(1,1)$ resonance (see section 2.1 for the meaning of $m, l)$ and the higher noneccentric resonances like $(m, l)=(2,2)$, while the eccentric binary will induce resonances like $(m, l)=(2,1),(3,1),(4,1)$ or higher harmonics. The resonance would lead to the tidal torque. When the total tidal torque at the edge of the disk is lager than the viscous torque, a gap is opened in the disk (Lin \& Papaloizou et al. 1986). The gap-opening condition for $(1,1)$ circular resonance is given by,

$$
\alpha^{1 / 2}\left(\frac{H}{r}\right) \leq 0.24 q(1-q)(1-2 q) .
$$

Here, $q$ is the mass ratio between the SMBBHs. The circumbinary disk around the SMBBHs can be described by $\alpha$ prescription ( Shakura \& Sunyaev et al. 1973). $r$ is the radius to the central mass point and $H$ is the corresponding scale height of the disk at radius $r$.

For the eccentric binary, the $(m, l)=(2,1),(3,1)$ resonance become more important. The gap-opening condition for $(2,1),(3,1)$ resonance is given by

$$
\alpha^{1 / 2}\left(\frac{H}{r}\right) \leq 1.76 e q(1-q)
$$

and 
respectively. Here $e$ is the eccentricity of the binary. If the values of $\alpha, \frac{H}{r}$ and $q$ are taken to be $0.05,0.01$ and 0.01 , the minimum eccentricity to open the $(2,1)$ and $(3,1)$ resonance gap is about 0.127 and 0.397 , respectively.

\subsection{Spectral energy distribution of the disk}

The disk is assumed to be in the local thermal equilibrium, that is, the heating and cooling are locally balanced at each point of the disk, $Q_{+}=Q_{-}$. The viscous heating term reads $Q_{+}=9 \Sigma \nu \Omega^{2} / 4$, where $\nu$ is the kinetic viscosity. The cooling term is given by the thermal emission: $Q_{-}=2 \sigma_{R} T_{\text {eff }}^{4}$, where $\sigma_{R}$ is the Stefan-Boltzmann constant, $T_{\text {eff }}$ is the effective temperature of the disk. So the effective temperature of each element of the disk is given by the equation:

$$
T_{\text {eff }}(r, \varphi)^{4}=\frac{9}{8} \frac{\nu}{\sigma} \Sigma(r, \varphi) \frac{G M}{r^{3}}
$$

The SED from an thermally emitting disk with the temperature as a function of the radius can be written as:

$$
F_{\lambda}=\int_{0}^{2 \pi} \int_{R_{\mathrm{in}}}^{R_{\mathrm{out}}} B_{\lambda}\left[T_{\text {eff }}\left(r^{\prime}, \varphi^{\prime}\right)\right] g\left(r^{\prime}\right) r^{\prime} d r^{\prime} d \varphi^{\prime},
$$

where $B_{\lambda}\left(T_{e f f}\right)$ is the Planck function and $g\left(r^{\prime}\right)$ is a function that describes the emissivity as a function of radius. We assume that $g\left(r^{\prime}\right)=1$ where the disk material is present and $g\left(r^{\prime}\right)=0$ elsewhere.

\section{PHYSICAL MODEL AND PARAMETERS}

We carry out simulation using the FARGO-ADSG code (Baruteau et al. 2008) in a two dimensional (R, $\phi$ ) fixed polar grid. FARGO-ADSG is an extended version of FARGO (Masset et al. 2000). In the FARGOADSG code, additional orbital advection which removes the average azimuthal velocity has been applied so that the truncation error is reduced and the time-step allowed by the Courant-Friedrichs-Lewy condition is significantly increased. The code was initially used to simulate the tidal interactions of planet-disk. We adopt it because of the similarity between the planet-disk and the SMBBHs-disk. The key model parameters are listed as follows:

Binary parameters - The mass of the primary and secondary black hole is taken to be $M_{1}=10^{8} M_{\odot}$ and $M_{2}=q M_{1}=4 \times 10^{6} M_{\odot}$, respectively. This is common in galaxy-dwarf galaxy minor mergers whose mass ratio $q$ ranges from 0.001 to 0.1 . In despite of the small mass ratio, recent numerical simulations have shown that the local processes can lead these binaries to be close enough (Bellovary et al.2010). The initial semi-major axis $a_{0}$ and eccentricity $e_{0}$ of the binary is taken to be $10^{3} r_{\mathrm{g}}\left(\approx 10^{-2} p c\right)$ and 0.5 , respectively. Disk parameters - Before its evolution, the disk is axisymmetric and rotates at Keplerian angular frequency $\Omega(r)$. The disk extends from $r=10 r_{\mathrm{g}}$ to $r=4 \times 10^{3} r_{\mathrm{g}}$ in our simulation. The mesh is $256 \times 156$ in the polar coordinates $(r, \theta)$. The initial gas density of the disk is about $6.6 \times 10^{5}\left(r / a_{0}\right)^{-1 / 2} \mathrm{~g} \mathrm{~cm}^{-2}$, which means that the initial disk mass is about $0.1 M_{1}$. The aspect ratio $h=H / r$ is assumed as 0.02 and remains constant throughout the simulation. In addition, the disk is modeled with a constant kinematic viscosity $\nu=2 \times 10^{15} \mathrm{~m}^{2} / \mathrm{s}$, which corresponds to an alpha viscosity $\alpha \approx 0.05$ at $0.01 \mathrm{pc}$. The criteria for gap opening is satisfied in our model.

The inner boundary condition in the code is chosen to be open such that material can flow outside of 


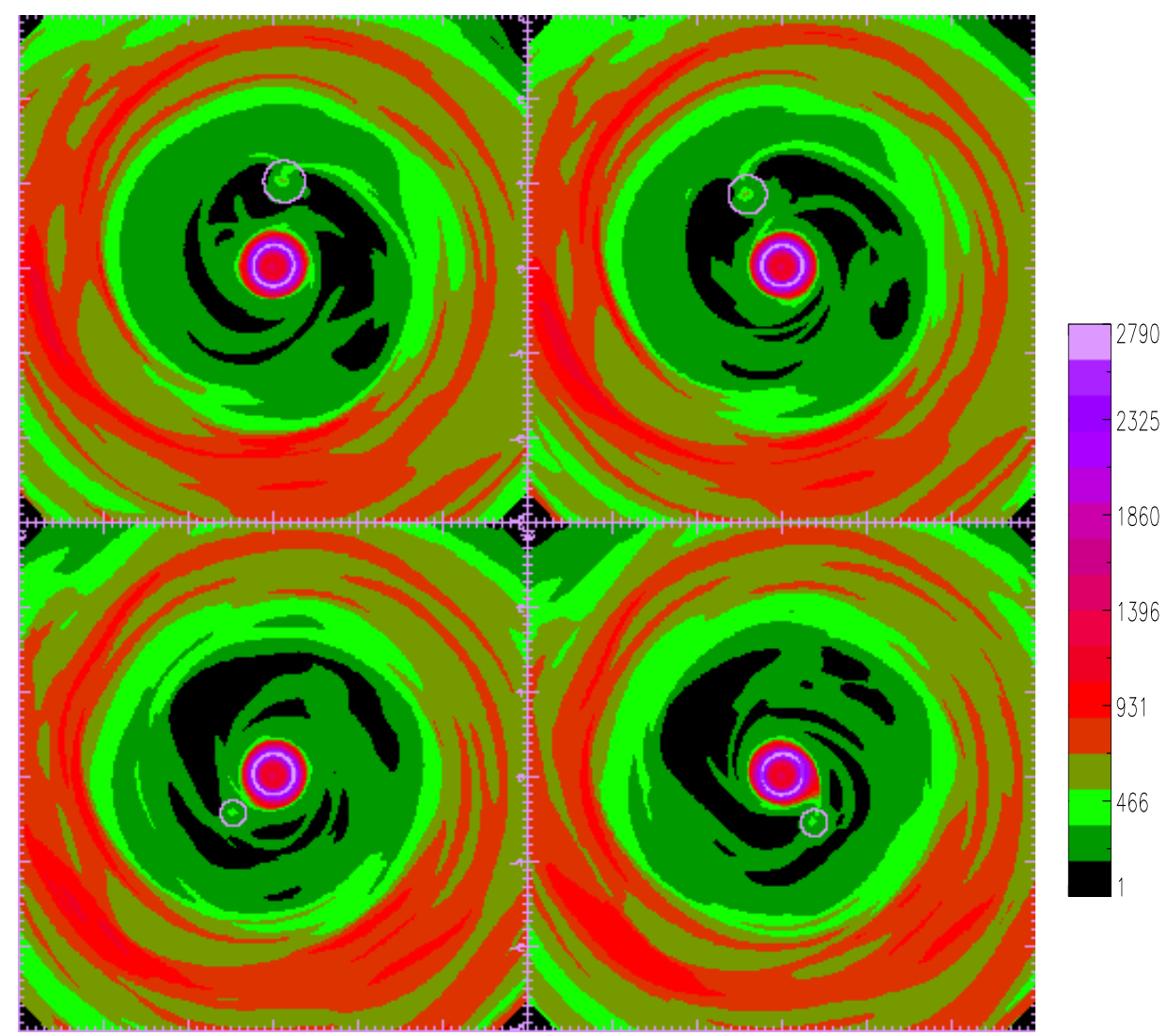

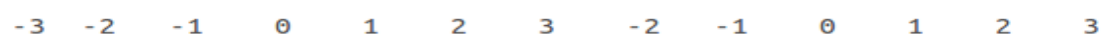

Fig. 1 Two-dimensional distributions of mass density at different orbital phases of the SMBBHs. Top left panel shows the result at apocenter phase. Bottom right panel shows the result at pericenter phase. Top right and Bottem left panels show two middle phases between the apocenter and pericenter phase, respectively. The plum circles represent the position of secondary BH. The primary black hole locates at the center, the coordinates are in units of $1000 R_{g}$ of the primary black hole. The density levels of each panel can be seen in the color bar on the right side. The black colour represents the minimum value of the surface density which is about $9.46 \times 10^{3} \mathrm{~g} \cdot \mathrm{cm}^{-2}$. The plum represents the maximum value which is 2790 times of the value of the black. The number of other colours shows the relative value of the surface density.

boundary condition which is not independent of the inner boundary condition can not be selected in the code.

\section{RESULTS AND DISCUSSIONS}

In this work, the simulation runs until a stable gap is opened in the circumbinary disk of SMBBHs. Our results are shown in Fig. 1-3.

We illustrate in Fig.1 the two-dimensional surface density distribution of the disk when the binary has a 


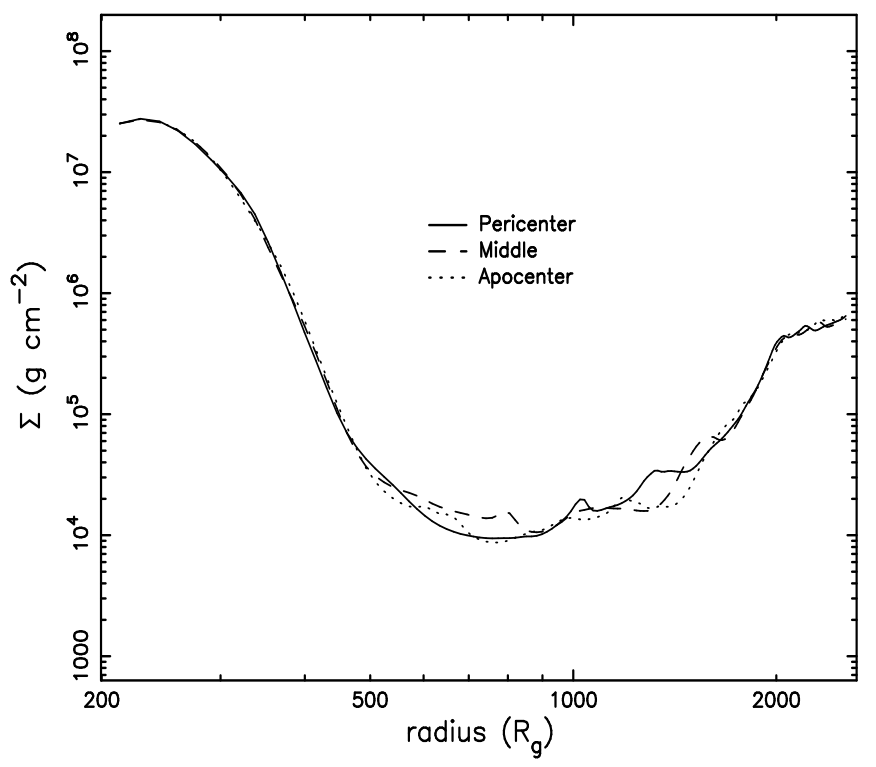

Fig. 2 One-dimensional distribution of the surface density within the radii between $220 R_{g}$ and $2600 R_{g}$.

the gap when it moves from the apocenter to the pericenter. Furthermore, a ring of high density exists in the inner disk, that can be attributed to the matter accumulation.

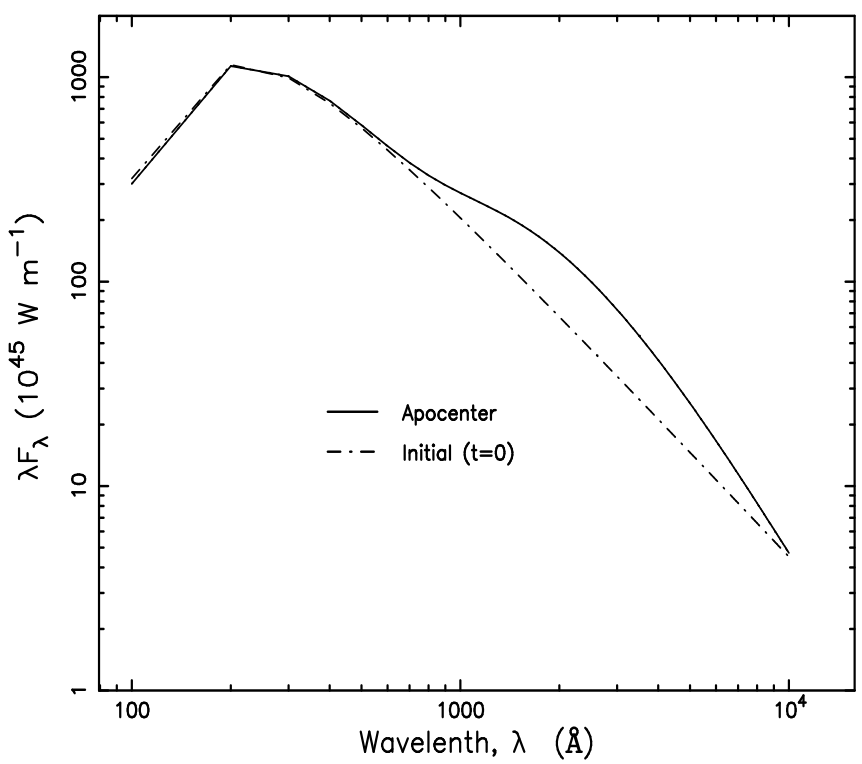

Fig. 3 Spectral energy distribution (SED) of the radiation from the accreting flow in the system of SMBBHs. After a stable gap is opened, solid line represents the SED at apocenter. The curves of SEDs at other orbital phases (middle and pericenter) vary little compared with that at apocenter and it is difficult to distinguish the curves at different orbital phases. The curves of SEDs at other orbital phases (middle and pericenter) are not plotted in this figure. For comparison, the initial SED is shown in the dot-dash line. At the initial moment, there is no gap in the disk. 
In order to clearly show the variation of the surface density, one-dimensional distribution of the surface density in one orbit is shown in Figure 2. The surface density varies significantly in the region of the gap (about $400 R_{g} \sim 1600 R_{g}$ ). The variation reaches $50 \%$ at $800 R_{g}$. However, the total amount of the gas crossing the gap is still too low to change the surface density of the inner disk (less than $1 \%$ below $400 R_{g}$ ).

It is meaningful to discuss the potential observational phenomena. Based on Equation (7) and (8), the emission fluxes at the various wavelengths are shown in Fig.3. There is little variation of the SED among the different orbital phases of the binary.

In this work we simulate the interaction between the eccentric SMBBHs and their circumbinary disk. Surface density of the disk evolves under the action of binary torques in one orbital period. The orbital period is $\sim 10 \mathrm{yr}$ in our model. For comparison, we estimate the timescale of viscosity (therefore the accretion timescale) near the outer edge of the inner disk (about $400 R_{g}$ ). The timescale of viscosity is $t_{v i s c} \sim R^{2} / \nu \sim 2.28 \times 10^{5} \mathrm{yr}$ with our model parameters. It is obvious that the accretion timescale is much longer than the orbital period. Therefore, the gas across the gap only accumulates at the outer boundary of the inner disk. It is impossible for the accumulated gas to be accreted into the central SMBH in one orbital period. Consequently, the surface density of inner disk does not vary significantly, as shown in Fig.2. The effective temperature in the disk is $T_{\text {eff }} \propto \Sigma^{1 / 4} / r^{3 / 4}$, so the effective temperature of the inner disk with larger surface density and smaller radius is higher than that of the other region. The emission from the inner disk with little variation dominates the thermal radiation of the disk. Therefore, the SED of the disk changes little as shown in Fig.3.

It is a natural idea to reduce the timescale of viscosity by increasing the value of the kinetic viscosity $\nu$. However, as we argue below, this will be contradictory to the criteria of the opening of a gap. Equation (5) must be satisfied to open a gap in the disk if we take (2:1) resonance gap as an example, which consequently results in a constraint on $\alpha$. The kinetic viscosity is $\nu=\alpha h^{2} a^{2} \Omega_{k}$, with the help of equation (5), we get $\nu \leq 3 e^{2} q^{2}(1-q)^{2} a^{2} \Omega_{k}$. Applying the typical parameters in our work $\left(e=0.3, q=0.04, a=0.01 p c, \Omega_{k}=\right.$ $\left.\sqrt{G M_{p} / a^{3}}=2.21 \times 10^{-8} \mathrm{rad} / \mathrm{s}\right)$, the kinetic viscosity at the binary radius satisfies $\nu \leq 8.55 \times 10^{17} \mathrm{~m}^{2} / \mathrm{s}$. Therefore, the typical viscosity timescale at the orbital radius $\left(t_{v i s c} \sim R^{2} / \nu \sim 3000 \mathrm{yr}\right)$ is at least 300 times longer than that of the binary period $(\sim 10 \mathrm{yr})$.

Recently, Bon (2012) has shown that a SMBBHs exists in the core of Seyfert galaxy NGC 4151. The periodic variations in the light curves and the radial velocity curves of NGC 4151 can be accounted for by an eccentric $(e=0.42)$, sub-parsec Keplerian orbit with a 15.9 year period. The periodic variations in the observed $H_{\alpha}$ line shape and flux are well explained in the model of the shock which is generated by the supersonic motion of the SMBBHs through the surrounding medium, rather than the periodic disk accretion. As we have argued that the periodic accretion of eccentric binary with sub-parsec separation does not cause obvious SED variation, this is consistent with the result of Bon (2012). As we adopt the $\alpha$ prescription of the disk in this work, it needs to be checked whether this prescription is still valid for the closed SMBBHs system. We hope that the future observations of SMBBHs candidates at sub-parsec separation will provide 
In this paper, the effect of GWs is neglected. We discuss the reason below. The timescale of orbital decay of the SMBBHs through GWs emission (Peters et al.1964) is

$$
t_{G W} \simeq 10^{4} \frac{(1+q)^{2}}{q}\left(\frac{10^{8} M_{\odot}}{M_{\text {total }}}\right)^{3}\left(\frac{a}{2 \times 10^{-3} p c}\right)^{4} \frac{1}{f(e)} y r .
$$

Here, $f(e)=\left(1+\frac{73 e^{2}}{24}+\frac{37 e^{4}}{96}\right)\left(1-e^{2}\right)^{-7 / 2}, e$ is the eccentricity of the binary system. $t_{G W}$ is about $8.8 \times 10^{7} \mathrm{yr}$ in our model and is much longer than the viscous timescale $\left(t_{v i s} \simeq 10^{6} \mathrm{yr}\right)$ at the orbital radius. Therefore it is reasonable to neglect the effect of GWs because of the domination of viscous effect.

The disk is modeled as a two-dimensional $(r, \varphi)$ system, by using the vertically averaged quantities. Two main arguments lie behind this choice. First, on a physical basis, the Hill radius of a massive object is large or comparable to the disk semi-thickness. Second, a less massive secondary SMBH has a weak impact on the disk, requiring a higher resolution to compute properly and highlight its effects. In the 2D simulation, it is more easy to increase the resolution of numerical simulation than in the 3D one. D'Angelo (2003) showed that two-dimensional simulations still yield reliable results when the mass ratio $q \geq 10^{-4}$. When the mass ratio $q<10^{-4}$, three-dimensional simulations are needed. The mass ratio is about $0.04(q=0.04)$ in our work, so the result might be still reliable.

Acknowledgements We would like to thank Prof. J.X. Wang for helpful discussions. This work is partially supported by National Basic Research Program of China (2009CB824800, 2012CB821800), the National Natural Science Foundation $(11073020,11133005,11233003)$, and the Fundamental Research Funds for the Central Universities (WK2030220004).

\section{References}

Artymowicz P., Lubow S., 1994, ApJ, 421, 651A

Baruteau C., Masset F., 2008, ApJ, 678, 483B

Begelman M. C., Blandford R.D. ,Rees M.J., 1980, Nature, 287, 307B

Bellovary J. M., Governato F., Quinn T. R., Shen S. J., Volonteri M., 2010, ApJ, 721L, 148B

Bogdanovic T., Reynolds C. S., Miller M. C., 2007, ApJ, 661L, 147B

Bon E., Jovanovic P., Marziani P., Shapovalova A., 2012, ApJ, 759, 118B

Comerford J. M., Griffith R. L., Gerke B. F., Cooper M. C., Newman J. A., Davis M., Stern D., 2009, ApJ, 702L, 82C

Colpi M., Dotti M., 2011, ASL, 4, 181

Crida A., 2009, ApJ, 698, 606

D’Angelo G., Kley W., Henning T., 2003, ApJ, 586, 540

Dotti M., Colpi M., Haardt F., 2006, MNRAS, 367, 103D

Dotti M., Volonteri M., Perego A., Colpi M., Ruszkowski M., Haardt F., 2010, MNRAS, 402, 682

Dotti M., Montuori C., Decarli R., Volonteri M., Colpi M., Haardt F., 2009, MNRAS, 398L, 73D

Espaillat C., Calvet N., Luhman K. L., Muzerolle J., Dalessio P., 2008, ApJ, 682L, 125E

Goldreich P., Tremaine S., 1980, ApJ, 241, 425G

Hayasaki K., Saito H., Mineshige S., 2012, preprint (astro-ph/1211.5137)

Hayasaki K., Mineshige S., Sudou H., 2007, PASJ, 59, 427H

Kley W., 1999, MNRAS, 303, 696

Komossa S., Burwitz V., Hasinger G., Predehl P., Kaastra J. S., Lkebe Y., 2003, ApJ, 582L, 15K

Komossa S., 2006, MmSAI, 77, 733K

Komossa S., Zhou H., Lu H., 2008, ApJ, 678L, 81K

Lin D.N.C., Papaloizou John., 1986, ApJ, 309, 846L

Liu X., Shen Y., Strauss M. A.,Green J. E., 2010, ApJ, 708, 427L 
Masset F., 2000, A\&AS, 141, 165M

Merritt D., Ekers R. D., 2002, Science,297, 1310

Peters P. C., 1964, Physics Review, 136, B1224

Rodriguez C., Taylor G. B., Zavala R. T., Peck A. B., Pollack L. K., Romani R. W., 2006, ApJ, 646, 49R

Roedig C., Dotti M., Sesana A., Cuadra J., Colpi M., 2011, MNRAS, 415, 3033

Sathyaprakash B.S., Schutz B. F., 2009, LRR, 12, 2 S

Shakura N. I., Sunyaev R. A., 1973, A\&A, 24, 337

Shen Y., Abraham L., 2010, ApJ, 725, 249S

Sudou H., Iguchi S., Murata Y., Taniguchi Y., 2003, Science, 300, 1263S

Takeuchi T., Miyama S. M., Lin D. N. C., 1996, ApJ, 460, 832T

Valtonen M. J. et al., 2008, Nature, 452, 851V

Wang J. M., Chen Y. M., Hu C., Mao W. M., Zhang S., Bian W. H., 2009, ApJ, 705L, 76W

Zhou H. Y., Wang T. G., Zhang X. G., Dong X. B., Li C., 2004, ApJ, 604L, $33 Z$ 